\title{
Time resolved anisotropic emission from an aluminium laser produced plasma
}

G. A. Wubetu, H. Fiedorowicz, J. T. Costello, and T. J. Kelly

Citation: Physics of Plasmas 24, 013105 (2017); doi: 10.1063/1.4973444

View online: https://doi.org/10.1063/1.4973444

View Table of Contents: http://aip.scitation.org/toc/php/24/1

Published by the American Institute of Physics

\section{Articles you may be interested in}

Time evolution analysis of dynamics processes in laser-produced Al plasmas based on a collisional radiative model

Physics of Plasmas 24, 013302 (2017); 10.1063/1.4973659

Temperature effect on femtosecond laser-induced breakdown spectroscopy of glass sample

Physics of Plasmas 24, 013301 (2017); 10.1063/1.4973658

Spatially resolved behavior of laser-produced copper plasma along expansion direction in the presence of static uniform magnetic field

Physics of Plasmas 23, 122104 (2016); 10.1063/1.4969080

Quantitative analysis of Al-Si alloy using calibration free laser induced breakdown spectroscopy (CF-LIBS)

Physics of Plasmas 24, 063516 (2017); 10.1063/1.4985327

Scattering cross sections of the particles in the partially ionized dense nonideal plasmas

Physics of Plasmas 24, 012101 (2017); 10.1063/1.4973324

Development of the dense plasma focus for short-pulse applications

Physics of Plasmas 24, 012702 (2017); 10.1063/1.4973227

\section{PHYSICS TODAY}

MANAGER'S GUIDE

WHITEPAPERS
READ NOW

PRESENTED BY

Accelerate R\&D with Multiphysics Simulation 


\title{
Time resolved anisotropic emission from an aluminium laser produced plasma
}

\author{
G. A. Wubetu, ${ }^{1,2, a)}$ H. Fiedorowicz, ${ }^{2}$ J. T. Costello, ${ }^{1}$ and T. J. Kelly ${ }^{1,3}$ \\ ${ }^{1}$ NCPST and School of Physical Science, Dublin City University, Glasnevin, Dublin 9, Republic of Ireland \\ ${ }^{2}$ Institute of Optoelectronics, Military University of Technology (MUT), Kaliskiego 2 Str. 00-908, Warsaw, Poland \\ ${ }^{3}$ School of Mathematics and Physical Sciences, University of Hull, Hull HU6 7RX, United Kingdom
}

(Received 14 August 2016; accepted 15 December 2016; published online 3 January 2017)

\begin{abstract}
The polarisation anisotropy of the emission from a laser produced aluminium plasma has been studied using time and polarisation resolved spectroscopy at various background pressures of air. A Wollaston prism was used to resolve the emission from the plasma into polarisation components that are parallel and orthogonal to the plasma expansion axis. Spectroscopy reveals that as the background pressure is increased, strongly polarised continuum emission dominates at early stages of the plasma formation. The results are compared and contrasted to similar experiments and discussed in the framework of a recombining plasma. Published by AIP Publishing.

[http://dx.doi.org/10.1063/1.4973444]
\end{abstract}

\section{INTRODUCTION}

The polarisation state of the light emitted from a laser produced plasma has recently become an area of interest in the wider laser plasma community. The reason for this is the observation that the continuum emission from a laser plasma tends to be more polarised than the line emission ${ }^{1-3}$ which is useful for analytical techniques such as Laser Induced Breakdown Spectroscopy (LIBS). In previous studies, the degree of polarisation $(\mathrm{P})$ of the light emitted from a laser produced plasma was measured for various focal positions, ${ }^{1,4}$ laser energies, ${ }^{4,5}$ background pressures, ${ }^{5,6}$ incident laser polarisations, ${ }^{3,4}$ detection directions, ${ }^{4,6}$ and pulse durations. ${ }^{1,6}$ In many cases, the use of a polariser in front of the spectrometer during a LIBS measurement suppressed the continuum and improved the limit of detection (LOD) by increasing the signal to background ratio. In one study, ${ }^{7}$ it was found that no significant polarisation of either the continuum or line emissions could be observed. This apparent null result compared with previous studies was resolved by taking the laser fluence into account. $^{2,6}$ In fact, this technique has birthed a subset of LIBS termed Polarisation-Resolved LIBS (PRLIBS).

The efficacy of this technique and the abundance of papers on the subject tend to belie the difficulty in understanding the creation of the partial polarisation of the emitted light. In a broader sense, Plasma Polarisation Spectroscopy (PPS) has existed since the 1920s when partial polarisation was observed in fluorescence experiments reported by Fujimoto and Kazantsev. ${ }^{8}$ The nature of the polarised emission is generally categorised into two classes:

(1) The electrons in the plasma do not follow a single Maxwell-Boltzmann distribution. This is sometimes called an anisotropic electron velocity distribution. ${ }^{5,9}$

(2) Self or external, electric, and magnetic fields exist which break the magnetic sub-level degeneracy. ${ }^{5}$

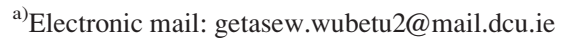

The early experiments on laser plasma polarisation spectroscopy date back to the mid-1990s, when the X-Ray spectrum of a highly charged aluminium plasma, produced with a picosecond laser, was found to exhibit a partial polarisation. ${ }^{9}$ It was found that the resonance and intercombination transitions are polarised differently. The polarisation of the intercombination lines was attributed to an anisotropic electron velocity distribution function arising from spatially anisotropic electron density profiles. Theoretically, the polarisation of recombination emission has been studied $^{10}$ and offered as an explanation for the polarisation of the emission. More recent experiments have observed the polarisation of lowly charged ions in aluminium ${ }^{11}$ in a low density and lowtemperature plasma, leading to the formation of ions in excited states which subsequently decay by spontaneous emission which is partially polarised due to an asymmetry in the magnetic sublevel populations in such excited states. The time-resolved polarisation of gas confined laser plasmas was studied, and a strong anisotropy was observed ${ }^{12}$ particularly for the case of short pulse irradiation (typically, during picosecond ablation). It was found in this study that the anisotropy of the emission from a particular Al III line $(569.6 \mathrm{~nm}$ wavelength) is stronger when the pulse duration is shorter. ${ }^{12}$ In fact, this is correlated to the electron-ion thermalisation time, and it was found that when the pulse duration is of the order of this time scale, the anisotropy is stronger.

In this work, we present a systematic study of the timeand polarised-resolved emissions from an aluminium laser plasma in different background environments. Emission spectra are measured simultaneously for plasma components, parallel and perpendicular to the quantization axis of the experiment (normal to the target). We measure the polarisation of different charge states of the aluminium plasma and correlate the degree of polarisation to the various radiation losses in the plasma. The results are discussed in the framework of class 1 and class 2 polarisation, as discussed earlier, and the efficacy of this technique for LIBS. 


\section{EXPERIMENTAL}

\section{A. Experimental setup}

Figure 1 shows the experimental setup used in this work. A Q-Switched Nd:YAG laser was focused onto an aluminium target to create a laser produced plasma. The laser used (Spectron SL800) had a 14 ns pulse duration and was operated at its fundamental wavelength of $1064 \mathrm{~nm}$. The fluence of the laser was varied using a combination of a halfwave plate and a polariser. For this experiment, the laser was focused normal to the target using a planoconvex lens ( $\mathrm{f}=250 \mathrm{~mm}$ ) onto a $\mathrm{Al}$ slab (purity $99.99 \%$ ) target to give a maximum fluence of $550 \mathrm{~J} / \mathrm{cm}^{2}$. The target was housed in a square vacuum sealed chamber which was evacuated at a pressure of $1 \times 10^{-5} \mathrm{mbar}$ and mounted onto a micrometre controlled $x-y-z$ translation stage which could be manipulated from outside the vacuum chamber. This ensured that a fresh target spot could be revealed after each laser shot. The plasma light emission was imaged normal to the laser propagation axis with a magnification of 0.5 using a two lens imaging system. A Wollaston prism was placed in the path of the imaging system to resolve the plasma image into two orthogonally polarised states. Thus, two images were formed in the image plane of the lens system. The optic axis of the Wollaston prism was aligned such that the plasma light was resolved into polarisation components parallel and perpendicular to the laser propagation direction. The plasma light was imaged onto the entrance slit of a Czerny-Turner spectrometer with a $1200 \mathrm{~g} / \mathrm{mm}$ grating and a focal length of $0.5 \mathrm{~m}$ giving an ultimate mean resolution of $0.17 \mathrm{~nm}$. At the exit of the spectrometer, the light was detected by a gated, intensified CCD camera (Andor i-Star) whose ultimate time resolution was $\simeq 10 \mathrm{~ns}$.

\section{B. Methodology}

Despite its usefulness, polarisation spectroscopy is somewhat obfuscated by many variable parameters. Thus, a clear methodology is required to interpret results correctly.

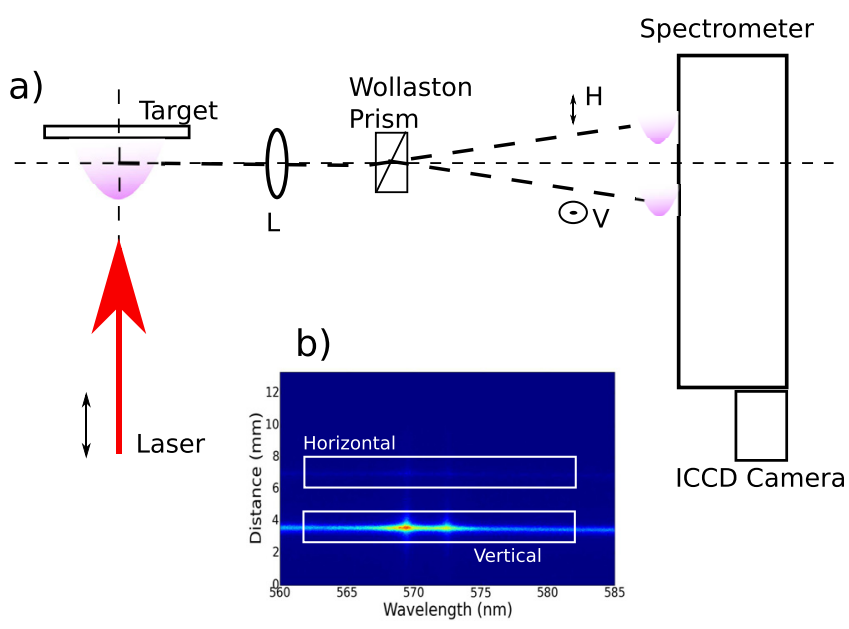

FIG. 1. Experimental setup (not to scale) showing the geometry of the experiment. A laser plasma is formed on a flat target. The plasma is imaged through a Wollaston Prism. Two plasma images are created on the entrance slit of an ICCD Camera. Inset is a typical image obtained by the experiment.
First, the accurate and precise values for the degree of polarisation can only be obtained if there exists no random intensity fluctuations between the polarisation components. A laser produced plasma will fluctuate naturally in intensity by $\simeq 10 \%$. Thus, making separate measurements of the different polarisation states limit the precision on the measurement. To account for this, the Wollaston prism method is used such that both polarisation states are measured in a single shot, eliminating the possibility of random or shot-to-shot fluctuation. To measure the precision and accuracy of our system, repeated measurements were made with an unpolarised light source. The standard deviation of the degree of polarisation was determined to be $\pm 0.01 \%$. Secondly, any measurement of polarisation must be done with respect to some reference axis, often called the axis of quantization. In our experiment, we use the following terminology: The plasma light was resolved into "Horizontal" and "Vertical" components. "Horizontal" refers to the direction normal to the target. "Vertical" refers to the direction parallel to the entrance slit of the spectrometer. It was determined that the efficiency of the ICCD camera and imaging system had no polarisation dependence in the wavelength ranges of interest to this study. However, the spectrometer was far more efficient for light polarised in the "Vertical" direction because this is also along the direction of the grating rulings. Normally, this is counteracted by normalising to some depolarised emission line (for example, the transition $4 s^{2} S_{1 / 2} \rightarrow 3 p^{2} P_{1 / 2}$ is unpolarised from a quantum mechanical point of view). ${ }^{5}$ However, line emission from a laser plasma tends to sit on top of continuum emission at the same wavelength. While the line emission from this transition is unpolarised, the continuum may in fact contain a partial polarisation. Thus, it is not always clear if the measurement can be normalised at this point. To account for the difference in efficiency for the different polarisations, emission from a xenon spectral lamp was polarised to $45^{\circ}$. This ensured that the intensity of the polarisation components at the entrance slit was equal. The measured difference through the spectrometer optical system was then used to calibrate further measurements.

The degree of polarisation at a particular wavelength $\left(P_{\lambda}\right)$ is the key parameter to measure the anisotropy emission from laser produced plasma. It is determined by the variance on the polarisation states. Mathematically, it is given by

$$
P_{\lambda}=\frac{I_{H}-I_{V}}{I_{H}+I_{V}},
$$

where $I_{H, V}$ are the intensities of the "Horizontal" and "Vertical" components respectively at a wavelength of $\lambda$.

The experiment was performed as follows. To benchmark the system, the time resolved value of $P_{\lambda}$ was measured with the PPS technique for the $A l^{2+}$ spectrum centred at $569 \mathrm{~nm}$. This was performed with a laser fluence of $550 \mathrm{~J} / \mathrm{cm}^{2}$ and a background pressure of $1 \times 10^{-5} \mathrm{mbar}$ to compare with similar experimental results. ${ }^{5}$ Air was then leaked into the chamber such that the base pressure rose to $1 \times 10^{-2} \mathrm{mbar}$ and the measurements were repeated. The experiment was then performed for the optical spectrum of $A l^{0}$ (centred at $395 \mathrm{~nm}$ ) at 

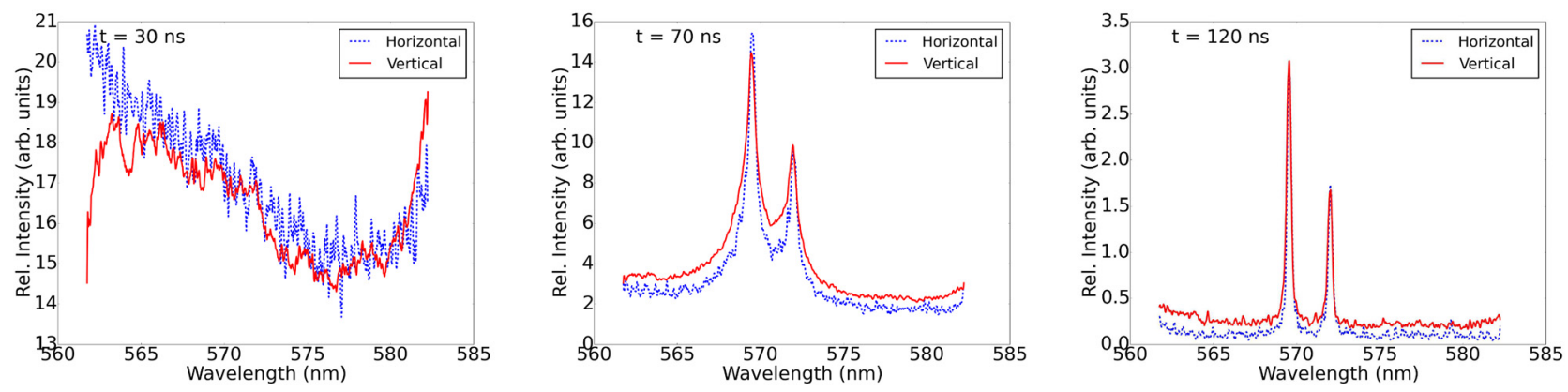

FIG. 2. Polarisation resolved spectra of $A l^{2+}$ for different stages of plasma evolution at a background pressure of $1 \times 10^{-2} \mathrm{mbar}$. The laser fluence was 550 $\mathrm{J} / \mathrm{cm}^{2}$ with an ICCD camera gate width of $10 \mathrm{~ns}$.
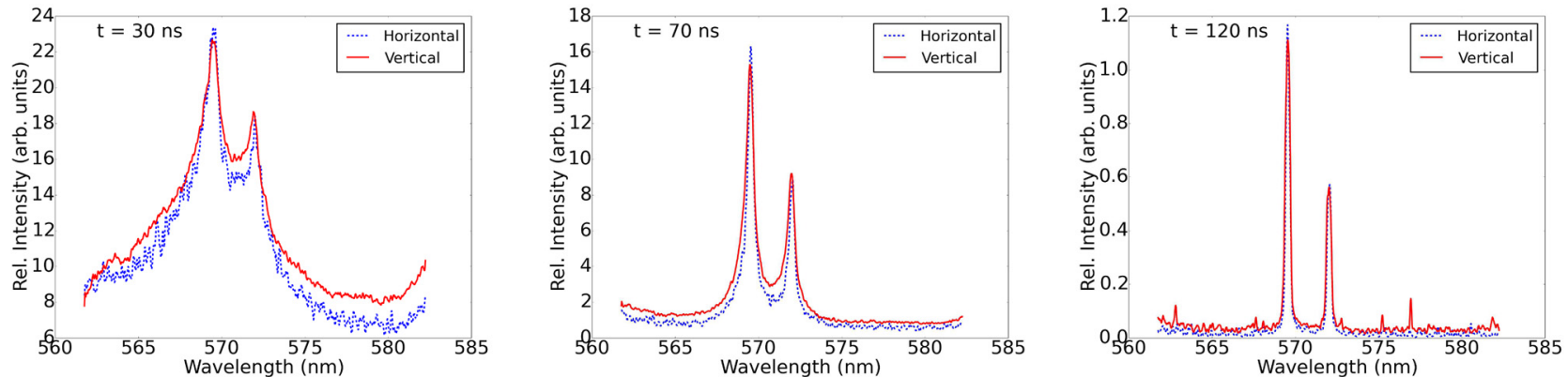

FIG. 3. Polarisation resolved spectra of $A l^{2+}$ for different stages of plasma evolution at a background pressure of $1 \times 10^{-5}$ mbar. The laser fluence was 550 $\mathrm{J} / \mathrm{cm}^{2}$ with an ICCD camera gate width of $10 \mathrm{~ns}$.

base pressures of $1 \times 10^{-2} \mathrm{mbar}$ and $1 \times 10^{3} \mathrm{mbar}$ at the same laser fluence. This gave a wide parameter space under which to compare and contrast the anisotropy of the laser plasma.

\section{RESULTS AND DISCUSSION}

\section{A. Spectroscopy results}

Figures 2 and 3 show polarisation resolved spectra at different stages of the plasma evolution for two different background pressures $\left(1 \times 10^{-2} \mathrm{mbar}\right.$ and $1 \times 10^{-5} \mathrm{mbar}$ respectively). In Figure 2, the spectrum is dominated by continuum emission. The later spectra are dominated by line emission. Figure 3 shows that as the pressure is lowered, the continuum emission dies away more quickly and the line structure of the spectrum appears sooner. As time progresses, for both background pressures, the plasma seems partially polarised mainly in the outer wings of the spectrum where the continuum emission is brightest. The degree of polarisation at $569 \mathrm{~nm}$ oscillates about zero as time proceeds. This is evidenced in Figure 4 where the degree of polarisation $\left(P_{\lambda}\right)$ was calculated using Equation (1) and plotted for both background pressures.

The degree of polarisation of the $A l^{2+}$ spectrum has been measured before in different contexts. ${ }^{5,11}$ The results in this study are in good agreement with these other reports. Previous reports ${ }^{5}$ have shown that $P_{\lambda}$ will be transient in nature and generally very small for the case of a plasma expanding into vacuum, which is in agreement with Figure 3. In previous reports, ${ }^{5}$ the degree of polarisation (of the $A l^{2+}$ line at $569 \mathrm{~nm}$ ) was increased with the background pressure and then become vanishingly small as the pressure was increased further. Figure 4 shows that as the background pressure is increased from $1 \times$ $10^{-5} \mathrm{mbar}$ to $1 \times 10^{-2} \mathrm{mbar}$, the degree of polarisation is, in general, higher. However, in this study, it was found that if the background pressure was increased to atmospheric pressure, the visible emission was dominated by singly charged ions and neutral emission. The polarisation resolved spectrum of $A l^{0}$ is shown at different stages of the plasma evolution at a background pressure of $1 \times 10^{-2}$ mbar as shown in Figure 5. The general trend is roughly in line with the spectrum of $\mathrm{Al}^{2+}$. No neutral emission was observed at

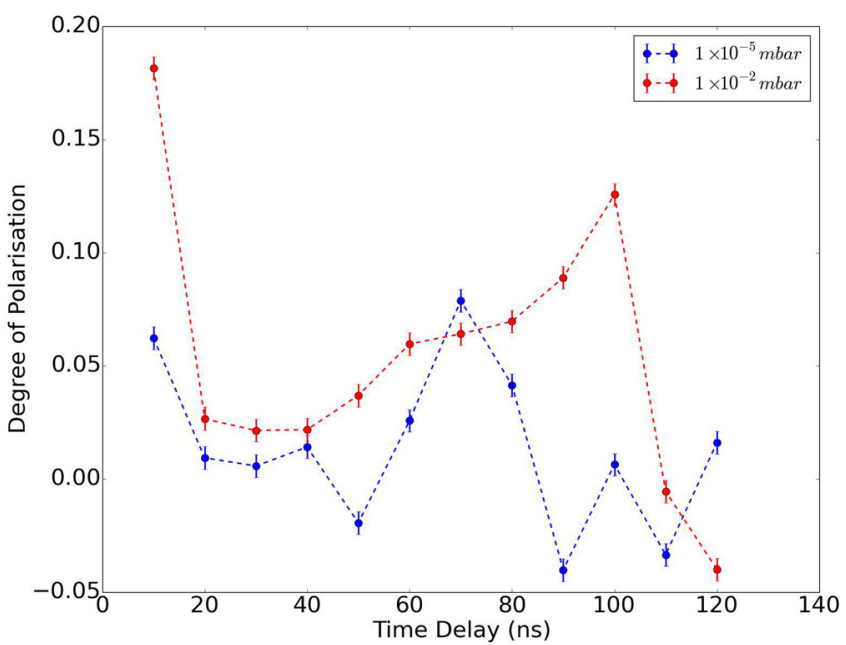

FIG. 4. Degree of polarisation calculated from the time resolved spectra in Figures 3 and 2. The wavelength of the spectrometer here was $569 \mathrm{~nm}$. 

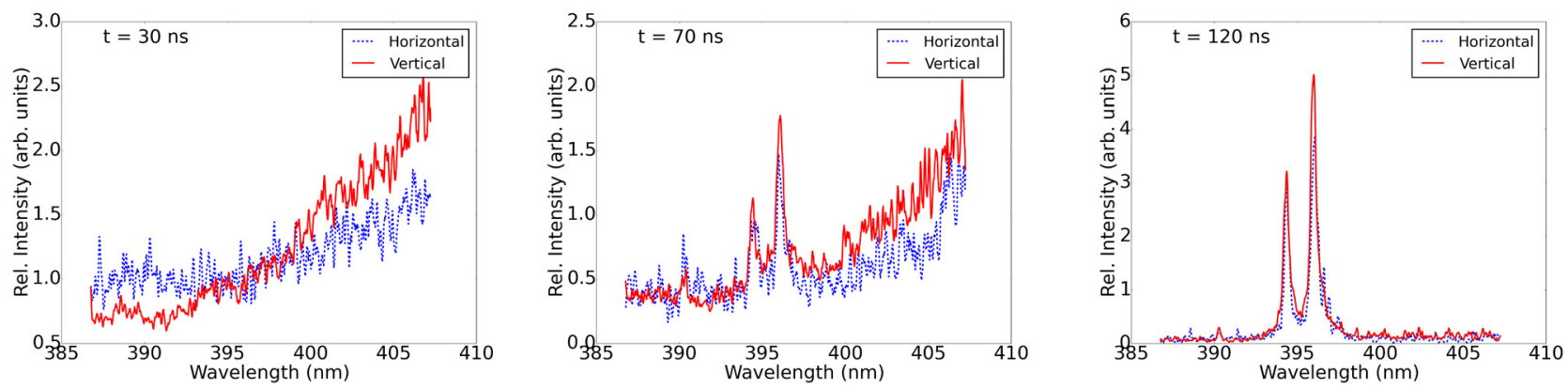

FIG. 5. Polarisation resolved spectra of $A l^{0}$ for different stages of plasma evolution at a background pressure of $1 \times 10^{-2}$ mbar. The laser fluence was 550 $\mathrm{J} / \mathrm{cm}^{2}$ with an ICCD camera gate width of $10 \mathrm{~ns}$.

$1 \times 10^{-5}$ mbar. The polarisation resolved spectrum of $A l^{0}$ is shown, in Figure 6, to be highly anisotropic and dominated by continuum emission during its evolution.

\section{B. Discussion}

The data presented would seem to indicate the following. First, as the background pressure is decreased, the spectrum becomes dominated by ionic emission over neutral emission, as is expected. Secondly, a higher background pressure is correlated with a longer lasting continuum spectrum. Thirdly, the line emission seems to be weakly polarised whereas the continuum emission seems to be more strongly polarised. This is seen not only in Figure 6 but also in Figure 3 where there is minimal polarisation in the spectrum, and it is mainly in the wings where the continuum emission is present. In the past, various mechanisms have been proposed to explain the anisotropic emission in laser plasmas including self generated magnetic fields, radiation trapping, and anisotropic electron distribution functions. It seems most appropriate here to describe the anisotropy as arising from radiative recombination in the laser plasma. For the same laser intensity, the initial and average temperatures of a plasma formed in vacuum and at atmospheric pressure should be similar. ${ }^{13}$ Thus, for a plasma in LTE, one would expect a similar charge state distribution present within the plumes. However, our observations indicate that the vacuum spectrum is dominated by highly charged $A l^{2+}$ ions and the atmospheric spectrum is dominated by the $A l^{0}$ emission. The long sustained continuum emission in the atmospheric case must then be due to forced recombination in the laser plasma which means the plasma is not in equilibrium because its radiative temperature is not equal to its kinetic temperature. This arises from the confinement of the plume in the background gas which causes free electrons to recombine with ions resulting in a lower average charge state. Another possibility lies in the fact that as the electrons in the atmospheric case try to expand away from the target, the plasma is stopped by the background gas. One would expect a planar expansion under atmospheric conditions and so the direction of deceleration of the electrons will be in the direction of expansion, i.e., along the target normal. In this case, the continuum emission would arise from this deceleration. If the deceleration of the electrons has a preferred direction, as we expect, then the emission should be polarised. This is one plausible explanation for the strong anisotropy in the atmospheric case. In the vacuum case, there is no background gas to confine the plume and so it expands and rapidly cools. The rates of free-free $\left(P_{f f}\right)$, free-bound $\left(P_{f b}\right)$, and bound-bound $\left(P_{b b}\right)$ emission are all temperature dependent in a laser plasma. They can be approximated as follows: ${ }^{14}$

$$
\begin{aligned}
P_{f f} & =1.5 \times 10^{-26} n_{e} T_{e}^{1 / 2} q^{2} n_{i}\left(\mathrm{Wm}^{-3}\right) \\
P_{f b} & =P_{f f} \frac{E_{I}}{T_{e}}\left(\mathrm{Wm}^{-3}\right) \\
P_{b b} & =P_{f f} \frac{2 \times 10^{7} e^{-\frac{E_{r}}{T_{e}}}}{T_{e} q^{2}}\left(\mathrm{Wm}^{-3}\right) .
\end{aligned}
$$

This explains the higher levels of continuum radiation in the plasma formed in atmosphere. In vacuum (taken here to mean $1 \times 10^{-5} \mathrm{mbar}$ ), the plasma expands which lowers its density $\left(n_{e}\right)$ thus lowering the value of $P_{f f}$ and $P_{f b}$. Eventually the
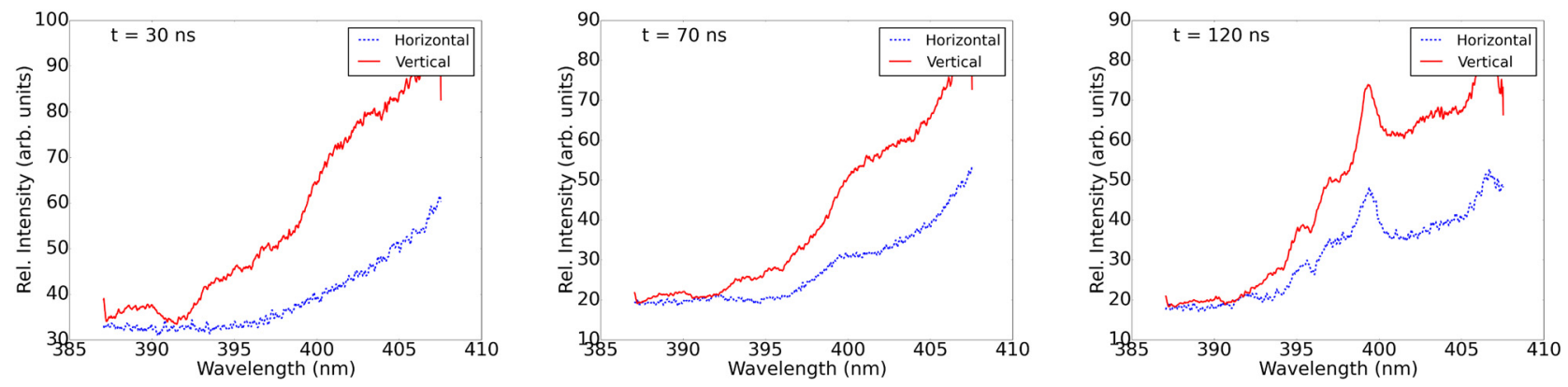

FIG. 6. Polarisation resolved spectra of $A l^{0}$ for different stages of plasma evolution at a background pressure of $1 \times 10^{3} \mathrm{mbar}$. The laser fluence was $550 \mathrm{~J} / \mathrm{cm}^{2}$ with an ICCD camera gate width of $10 \mathrm{~ns}$. 
radiative losses lower the electron temperature $\left(T_{e}\right)$ to a value close to the resonance energy for a particular transition in an atom or ion in the plasma, increasing the value of $P_{b b}$. This explains the dominance of ionic line emission under vacuum. For a plasma formed in air, the confinement causes a higher $n_{e}$ value which increases the rate of $P_{f f}$ and hence $P_{f b}$. Thus, it can be concluded that, for any given time, the continuum radiation power should scale with the background pressure. It is now perhaps useful to consider whether the rate of $P_{f f}$ is higher or lower than the rate of $P_{f b}$. Equation (2) shows that if the electron temperature $T_{e}$ is lower than the ionization energy of the bound state to which the electron is captured $\left(E_{I}\right)$, then the radiative recombination will be preferred. Under these experimental conditions, temperature analysis is not possible by optical spectroscopy, however, it can be inferred that just after ablation, radiative recombination is the main energy dissipation channel in the plasma. Detailed frequency and time resolved measurements of plasmas under similar experimental conditions to this work have shown that the preferential energy dissipation method just after laser heating will be radiative recombination which populates the lower charged ions and neutral atoms. ${ }^{15}$ Thus, to explain the results here, the model of anisotropic emission from radiative recombination can be used. Previous theoretical analysis has shown that the degree of polarisation of the recombination emission is inversely proportional to the electron temperature in the plasma. ${ }^{1,10}$ This explains why the degree of polarisation is generally higher in the atmospheric case. The high density leads to a higher level of continuum radiation from free-free and radiative recombination processes. The adiabatic and radiative losses lower the plasma temperature and increase the value of the anisotropy. Similarly, the plasma confinement due to the background gas sustains the electron density, the continuum radiation, and therefore the polarisation.

It is perhaps wise to make some mention as to the sign of the polarisation in both Figures 4 and 7. In general, the polarisation is generally positive in Figure 4 and negative in Figure 7. One possible explanation for this is to consider the total polarisation of the emission at a specific wavelength as being the combination of anisotropy from two sources: (a) the

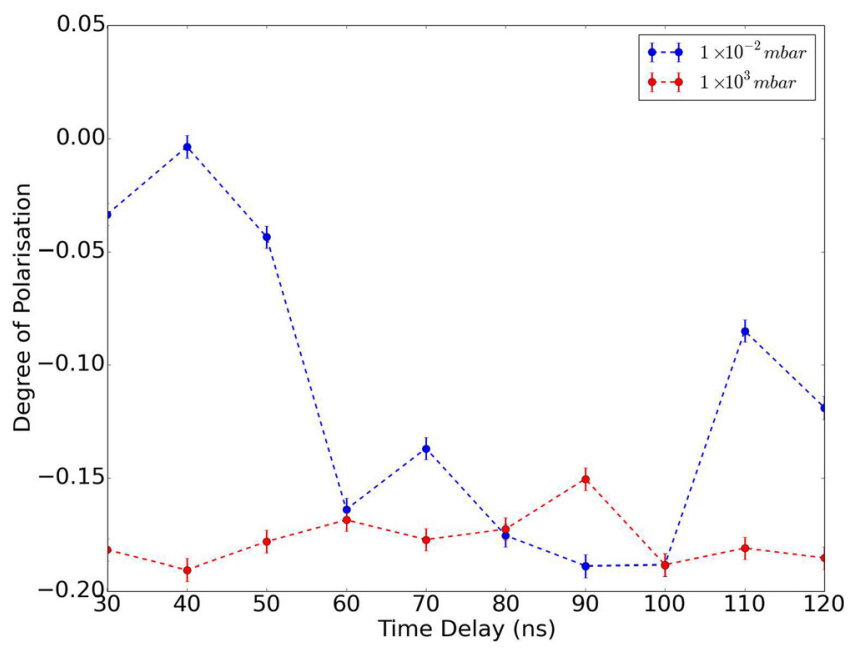

FIG. 7. Degree of polarisation calculated from the time resolved spectra in Figures 5 and 6 . The wavelength of the spectrometer here was $395 \mathrm{~nm}$. bound-bound emission and (b) the free-free or free-bound emission. The direction of the free-free or free-bound polarisation is along the expansion axis of the plasma, which, in our experimental scheme, is a negative polarisation direction. The second source of polarisation comes from the boundbound emission. The polarisation of this emission arises from the angular momentum conservation in transitions between magnetic sub levels which have a statistical imbalance in their populations. The direction of this polarisation should be perpendicular to the quantisation axis of the experiment, which in our scheme is along the expansion axis. This results in a positive polarisation. Hence the overall direction of polarisation at a particular wavelength/time/pressure is dependent on whether the continuum of line emission dominates.

There is empirical evidence from our data to support this as a valid hypothesis. Perhaps it is most readily seen by looking at Figure 3, middle panel $(t=70 \mathrm{~ns})$. Focussing on a wavelength of $569 \mathrm{~nm}$, it is clear the polarisation is in the positive direction (Horizontal $>$ Vertical). However, looking at something like $565 \mathrm{~nm}$, the polarisation is negative (Vertical $>$ Horizontal). We believe this is due to the fact that under the conditions that the spectrum was taken, the line emission dominates the emission at the $569 \mathrm{~nm}$ wavelength under consideration here. In cases where the continuum emission dominates, the polarisation should be negative.

Regarding the efficacy of this technique for LIBS, the data here would seem to indicate that the PRLIBS technique is effective at suppressing the background continuum when radiative recombination is the dominant radiation loss process. This appears to be true for the atmospheric but not for the vacuum case. The power of the recombination process depends on the experimental parameters such as plasma temperature and density and background pressure. Similarly, the continuum radiation appears to be sustained for a longer time in the atmospheric case. This is due to confinement of the plume by the background gas. Again, the duration of this sustained radiation and hence the duration of the times during which there is large anisotropy will be dependant on experimental parameters. PRLIBS is often touted as an advantageous alternative to gated-LIBS due to the fact that the parameters of gated-LIBS are tied to the experimental conditions. ${ }^{4}$ It would appear from this study that PRLIBS also depends on the experimental conditions and therefore care must be taken when using this technique effectively. Similarly, our data indicate that the origin of the polarisation can be explained without the need to consider the target experimental geometry. It is most likely the case, then, that the totality of the polarised emission results from a combination of sources such as: polarisation of line emission, ${ }^{11}$ experimental configuration (such as Fresnel reflection resulting from oblique laser incidence on the surface) ${ }^{16}$ and recombination emission and is unlikely to be explained by any single mechanism.

\section{CONCLUSIONS}

In this study, the time and polarisation resolved spectroscopy of an aluminium laser produced plasma was performed. 
The results indicate that the plasma has a high degree of polarisation at early times during its evolution and that this scales with the background pressure. From rate equations for various radiation losses in the plume, it is inferred that the continuum radiation should dominate at high background pressures. Similarly, conclusions from a theoretical model for anisotropy in radiative recombination emission seems to describe the trends found in this data. Thus, we present a strong evidence that the anisotropy in the plasma plume is driven by radiative recombination during the early phase of the plume, when the plasma is out of LTE.

\section{ACKNOWLEDGMENTS}

The work is supported by the Education, Audio-visual and Culture Executive Agency (EACEA) Erasmus Mundus Joint Doctorate Programme EXTATIC, Project No. 2013 0033 and Science Foundation Ireland Grant Nos. 12/IA/1742 and 14/TIDA/1742. The work is associated with COST Action MP1208 and U.S. National Science Foundation PIRE Grant No. 1243490.

\footnotetext{
${ }^{1}$ N. Agnes, Z.-Q. Hao, J. Liu, H.-Y. Tao, X. Gao, C.-K. Sun, and J.-Q. Lin, "The high dependence of polarization resolved laser-induced breakdown spectroscopy on experimental conditions," Chin. Phys. B 21, 074204 (2012).

${ }^{2}$ L. Jia, T. Hai-Yan, Gao Xun, H. Zuo-Qiang, and L. Jing-Quan, "The polarization characteristics of single shot nanosecond laser-induced breakdown spectroscopy of Al," Chin. Phys. B 21, 014209 (2012).

${ }^{3}$ A. Eslami Majd, A. S. Arabanian, and R. Massudi, "Polarization resolved laser induced breakdown spectroscopy by single shot nanosecond pulsed Nd:YAG laser," Opt. Lasers Eng. 48, 750-753 (2010).
}

${ }^{4}$ J. S. Penczak, Y. Liu, and R. J. Gordon, "Polarization resolved laserinduced breakdown spectroscopy of Al," J. Phys. Chem. 113, 13310-13317 (2009).

${ }^{5}$ A. K. Sharma and R. K. Thareja, "Anisotropic emission in laser-produced aluminum plasma in ambient nitrogen," Appl. Surf. Sci. 253, 3113-3121 (2007).

${ }^{6}$ D. Zhao, N. Farid, R. Hai, D. Wu, and H. Ding, "Diagnostics of first wall materials in a magnetically confined fusion device by polarizationresolved laser-induced breakdown spectroscopy," Plasma Sci. Technol. 16, 149-154 (2014).

${ }^{7}$ M. E. Asgill, H. Y. Moon, N. Omenetto, and D. W. Hahn, "Investigation of polarization effects for nanosecond laser-induced breakdown spectroscopy," Spectrochim. Acta - Part B At. Spectrosc. 65, 1033-1040 (2010).

${ }^{8}$ T. Fujimoto and S. A. Kazantsev, "Plasma polarization spectroscopy plasma polarization spectroscopy review article," Plasma Phys. Controlled Fusion 39, 1267 (1997).

${ }^{9}$ J. C. Kieffer, J. P. Matte, M. Chaker, Y. Beaudoin, C. Y. Chien, S. Coe, G. Mourou, J. Dubau, and M. K. Inal, "X-ray-line polarization spectroscopy in laser-produced plasmas,” Phys. Rev. E 48, 4648-4658 (1993).

${ }^{10}$ H. M. Milchberg and J. C. Weisheit, "Polarization of recombination radiation from nonequilibrium plasmas," Phys. Rev. A 26, 1023-1029 (1982).

${ }^{11}$ J. Kim and D. Kim, "Measurement of the degree of polarization of the spectra from laser produced recombining Al plasmas," Phys. Rev. E Stat., Nonlinear, and Soft Matter Phys. 66, 017401 (2002).

${ }^{12}$ A. K. Sharma and R. K. Thareja, "Polarization-resolved measurements of picosecond laser-ablated plumes,'” J. Appl. Phys. 98, 033304 (2005).

${ }^{13} \mathrm{~B}$. Wu, "High-intensity nanosecond-pulsed laser-induced plasma in air, water, and vacuum: A comparative study of the early-stage evolution using a physics-based predictive model," Appl. Phys. Lett. 93, 101104 (2008).

${ }^{14}$ M. J. Bernstein and G. G. Comisar, "X-ray production in laser-heated plasmas,” J. Appl. Phys. 41, 729-733 (1970).

${ }^{15}$ G. Bertuccelli, H. Di Rocco, D. Iriarte, M. R. Bidegrain, and H. RaneaSandoval, "Radiative mechanisms in laser-produced plasmas in Xe," J. Quant. Spectrosc. Radiat. Transfer 61, 309-317 (1999).

${ }^{16}$ J. S. Penczak, Y. Liu, R. D. Schaller, D. H. Rich, and R. J. Gordon, "The mechanism for continuum polarization in laser induced breakdown spectroscopy of Si(111)," Spectrochim. Acta - Part B At. Spectrosc. 74-75, 3-10 (2012). 